\title{
BRAIDS, LINK POLYNOMIALS AND A NEW ALGEBRA
}

\author{
JOAN S. BIRMAN AND HANS WENZL
}

\begin{abstract}
A class function on the braid group is derived from the Kauffman link invariant. This function is used to construct representations of the braid groups depending on 2 parameters. The decomposition of the corresponding algebras into irreducible components is given and it is shown how they are related to Jones' algebras and to Brauer's centralizer algebras.
\end{abstract}

In [J,3] Vaughan Jones announced the discovery of a new polynomial invariant of knots and links, which bore many similarities to the classical Alexander polynomial, but was seen to detect properties of a link which could not be detected by the Alexander invariants. The discovery was a real surprise, one of those exciting moments in mathematics when two seemingly unrelated disciplines turn out to have deep interconnections. The discovery came about in the following way. Jones' earlier contributions in the area of Operator Algebras had produced, in [J,1], a family of algebras $A_{n}(t), t \in \mathbf{C}$, indexed by the natural numbers $n=1,2,3, \ldots$, and equipped with a trace function $\tau: A_{n}(t) \rightarrow \mathrm{C}$. His algebra $A_{n}(t)$ was a quotient of the well-known Hecke algebra of the symmetric group, which we denote by $\mathscr{H}_{n}(l, m)$ to delineate our particular 2-parameter version of it. Jones had discovered, in [J,2], that there were representations of Artin's braid group $B_{n}$ in the algebra $A_{n}(t)$, in fact there were maps

$$
B_{n} \stackrel{\phi}{\longrightarrow} \mathscr{H}_{n}(l, m) \stackrel{\rho_{2}}{\longrightarrow} A_{n}(t)
$$

from $B_{n}$ into the multiplicative group of $A_{n}(t)$ which factored through $\mathscr{H}_{n}(l, m)$.

Links enter the picture via braids. Each oriented link $L$ in oriented $S^{3}$ can be represented by a (nonunique) element $\beta$ in some braid group $B_{n}$. There is an equivalence relation on $B_{\infty}=\coprod_{n=1}^{\infty} B_{n}$, known as Markov equivalence, which determines a 1-1 correspondence between equivalence classes $[\beta] \in B$ and isotopy types of the associated oriented links $L_{\beta}$. Jones' discovery was that with a small renormalization his trace function on $A_{\infty}(t)=\coprod_{n=1}^{\infty} A_{n}(t)$ could be made into a function which lifted to an invariant on Markov classes

Received by the editors December 9, 1987.

1980 Mathematics Subject Classification (1985 Revision). Primary 57M25; Secondary 20F29, $20 \mathrm{C} 07$.

The work of the first author was supported in part by NSF grant \#DMS-8503758.

The work of the second author was supported in part by NSF grant \#DMS-8510816. 
in $B_{\infty}$. That modified trace, described in [ $\left.\mathrm{J}, 3\right]$ and in more detail in $[\mathrm{J}, 4]$, is the Jones polynomial $V_{L}(t)$. (It becomes a polynomial when the parameter $t$ is regarded as an indeterminate.)

The polynomial $V_{L}(t)$ was quickly generalized in a six-author paper [FYHLMO], to a 2-variable polynomial $P_{L}(l, m)$. One of the authors was A. Ocneanu. Ocneanu's interpretation of $P_{L}(l, m)$, as described in [FYHLMO] and $[\mathrm{J}, 4]$, is via a lift of the Jones trace to the Hecke algebra $\mathscr{H}_{n}(l, m)$. Others, notably Lickorish and Millett [L-M] and Hoste [H], had discovered the identical polynomial $P_{L}(l, m)$ by combinatorial methods which had little to do with braids, traces or algebras.

On the heels of $P_{L}(l, m)$ came two additional polynomials, the Kauffman polynomial $K_{L}(l, m)$ and a precurser later identified as $K_{L}(l, m)$. References are [B-L-M and $\mathrm{K}, 1]$. These were proved to be well defined by various combinatorial methods, including not only generalizations of the methods used in [L-M and $\mathrm{H}$ ], but also new techniques in [K,1], which again bypassed braids. The polynomials $P_{L}(l, m)$ and $K_{L}(l, m)$ were shown to be independent, with each distinguishing links the other could not distinguish. On the other hand (see [L] $), V_{L}(t)=K_{L}\left(t^{-3 / 4},-\left(t^{1 / 4}+t^{-1 / 4}\right)\right)=P_{L}\left(i t^{-1},-i\left(t^{1 / 2}-t^{-1 / 2}\right)\right)$. (We shall have more to say about this curious fact in $\S 4$ below.)

The purpose of this note is to reverse the process begun by Jones. We will use the existence of $K_{L}(l, m)$, and apply the methods used to construct it in $[\mathrm{K}, 1]$ to construct a new two-parameter family of finite-dimensional algebras, $\left\{\mathscr{C}_{n}(l, m) ; n=1,2,3, \ldots\right\}$, complete with trace, such that $K_{L}(l, m)$ is, after appropriate renormalization, that trace, just as $P_{L}(l, m)$, renormalized, was shown by Ocneanu to be the trace on $\mathscr{H}_{n}(l, m)$.

In $\S 1$ below, we will review the background. In $\S 2$ we define our algebra by generators and relations, and explain our motivation, which is based upon Kauffman's work. In $\S 3$ we study the algebra $\mathscr{C}_{n}(l, m)$. We prove that $\mathscr{C}_{\infty}(l, m)=$ $\amalg_{n \in \mathbf{Z}}^{\infty} \mathscr{C}_{n}(l, m)$ supports a nondegenerate trace. The existence of this trace is a direct consequence of the fact that $K_{L}(l, m)$ is a link type invariant. We use the existence of the trace to uncover the structure of $\mathscr{C}_{n}(l, m)$, by techniques which derive from the "basic construction" of Jones in $[\mathrm{J}, 1]$. We prove that $\mathscr{C}_{n}(l, m)$ is semisimple and is a direct sum $\mathscr{H}_{n} \oplus \mathscr{H}_{n}^{\prime}$, where $\mathscr{H}_{n}=\mathscr{H}_{n}(l, m)$. The structure of $\mathscr{H}_{n}^{\prime}$ as an algebra over $\mathbf{C}[l, \alpha]$, where $m=\alpha+\alpha^{-1}$, is determined inductively from the inclusion pattern $\mathscr{C}_{n-2} \subset \mathscr{C}_{n-1}$. The dimension of $\mathscr{H}_{n}(l, m)$ is $n$ ! (see [J,4] for a proof of this well-know fact). It follows from our structure theorem that the dimension of $\mathscr{C}_{n}(l, m)$ is $1 \cdot 3 \cdot 5 \cdots(2 n-1)$.

The relationship between $\mathscr{C}_{n}(l, m)$ and the 1-parameter Jones algebra $A_{n}(t)$ is very interesting. We prove in $\S 4$ that there are two distinct homomorphisms from $\mathscr{C}_{n}(l, m)$ onto $A_{n}(t)$. One of them factors through $\mathscr{H}_{n}(l, m)$, and is not unexpected in view of the fact that $\mathscr{C}_{n}(l, m)=\mathscr{H}_{n} \oplus \mathscr{H}_{n}^{\prime}$, with $\mathscr{H}_{n}(l, m)$ mapping in a known way onto $A_{n}(t)$. The other does not factor through $\mathscr{H}_{n}(l, m)$. The manner in which the irreducible representations of $\mathscr{C}_{n}(l, m)$ go over to 
irreducible representations of $A_{n}(t)$ in the second homomorphism seems quite remarkable. We discovered that there are two homomorphsims in our attempts to understand the curious fact that $V_{L}(t)$ occurs both as $P_{L}\left(i t^{-1}, i\left(t^{1 / 2}-t^{-1 / 2}\right)\right)$ and as $K_{L}\left(t^{-3 / 4},-t^{1 / 4}-t^{-1 / 4}\right)$.

The existence of these two homomorphisms is not the only curious fact about $\mathscr{C}_{n}(l, m)$. In $\S 5$ we discuss an algebra which was studied by R. Brauer in 1937 [Br]. Like $\mathscr{C}_{n}(l, m)$, Brauer's algebra has dimension $1 \cdot 3 \cdot 5 \cdots(2 n-1)$. It is defined by pictures which bear a striking similarity to our pictures, indeed his pictures give a very easy way to find a basis for our algebra. We will show that our algebras can be regarded as a deformation of Brauer's similarily as $\mathscr{H}_{n}(l, n)$ is a deformation of $\mathbf{C} S_{n}$. This was also observed independently by H. Morton and P. Traczyk (see $[M T]$ ).

The final section of the paper, $\S 6$, discusses a possible application of our work to the question of whether the braid group is a linear group.

\section{ACKNOWLEDGEMENT}

The definition of our algebra $\mathscr{C}_{n}(l, m)$ in $\S 2$ below was motivated by pictures of a certain monoid which generalizes Artin's braid group $B_{n}$. The monoid has been used by various authors, although to our knowledge it remains a bit mysterious at this writing. The first author first learned of this monoid years ago from Claude Bourin [Bo], and again more recently had discussions about it with David Yetter [Y]. It has figured most recently in the work of Kauffman $[\mathrm{K}, 1$ and $\mathrm{K}, 2]$.

We first learned about Brauer's algebra from Vaughan Jones. We thank him for telling us about it, and for his continuing lively interest in this work. We also wish to thank Phillip Hanlon for telling us many interesting things about the Brauer algebra.

Related results have been obtained simultaneously and independently by J. Murakami [M], who also constructs an algebra from Kauffman's polynomial duplicating our $\S 2$, but not our $\S \S 3-6$.

\section{The bRaid gRoup $B_{n}$, THE AlgebRA $\mathscr{H}_{n}$, AND}

THE LINK POLYNOMIAL $P_{L}(l, m)$

Let $\vec{p}=\left(p_{1}, \ldots, p_{n}\right)$ be an $n$-tuple of distinct points $p_{i}$ on the complex plane, which for convenience may be assumed to lie along the $x$-axis. For present purposes, a braid $\beta$ on $n$-strands is an equivalence class $[e]$ of levelpreserving embeddings of $n$ disjoint copies of the unit interval $I^{(n)}=\coprod_{j=1}^{n} I_{j}$ in $\mathbf{C} \times I$, where $e$ sends the $n$ copies of $\{0\}$ (respectively $\{1\}$ ) to $\vec{p} \times\{0\}$ (respectively $\vec{p} \times\{1\}$ ). The equivalence relation is $e \approx e^{\prime}$ if $e$ is isotopic to $e^{\prime}$ rel $\partial I^{(n)}$. Multiplication is by concatenation and rescaling, and the identity element is the constant embedding $e_{j}(t)=p_{j} \times t$ for each $t \in I_{j}$, where $e_{j}$ is the $j$ th coordinate function, $j=1, \ldots, n$. This makes the set of all $n$-braids into a group $B_{n}$, Artin's braid group. A typical element of $B_{4}$ is illustrated in 
Figure 1, via its projection onto the $x-t$ plane, but with double points replaced by overpasses and underpasses. From such pictures it is intuitively clear that the elementary braids $\sigma_{1}, \ldots, \sigma_{n-1}$ shown in Figure 2 generate $B_{n}$. This was proved by Artin in [Ar], who also proved that defining relations are:

$$
\begin{gathered}
\sigma_{i} \sigma_{j}=\sigma_{j} \sigma_{i} \quad \text { if }|i-j| \geq 2,1 \leq i, j \leq n-1 ; \\
\sigma_{i} \sigma_{i+1} \sigma_{i}=\sigma_{i+1} \sigma_{i} \sigma_{i+1}, \quad 1 \leq i \leq n-2 .
\end{gathered}
$$

Note that there is a natural way to orient a braid, determined by the orientation on the unit interval. We orient our braids from top to bottom.

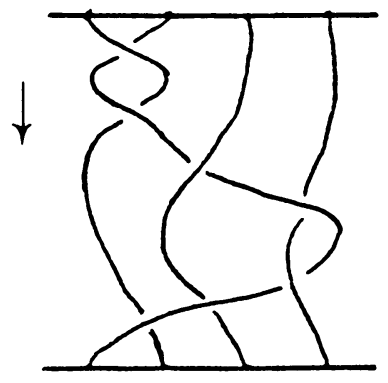

FIGURE 1

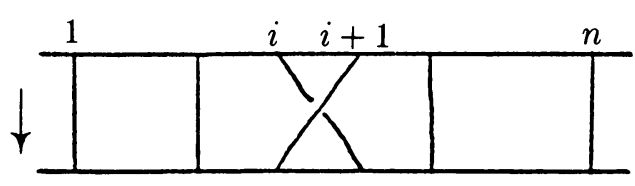

FIGURE 2

Links are obtained from braids when the free ends at the top and bottom of a braid are joined up by $n$ disjoint arcs as in Figure 3 to form a closed braid. If $\beta \in B_{n}$, we denote the link so-obtained by $L_{\beta}$. It carries a natural orientation, determined by the orientation on $\beta$.

Two oriented links $L, L^{\prime}$ in oriented 3-space are equivalent if $L$ is isotopic to $L^{\prime}$. The equivalence class is a link type. Alexander proved in [A1] that every oriented link is isotopic to $L_{\beta}$ for some (nonunique) element $\beta \in B_{\infty}$, $B_{\infty}=\coprod_{n=1}^{\infty} B_{n}$. Markov's theorem (see [B]) asserts that the equivalence relation on $B_{\infty}$ which is generated by the following two moves:

(i) $\beta \leftrightarrow \alpha \beta \alpha^{-1}, \alpha, \beta \in B_{n}$;

(ii) $B_{n} \leftrightarrow B_{n+1}$, by $\beta \leftrightarrow \beta \sigma_{n}^{ \pm 1}$

determines the equivalence class of all elements $\beta \in B$ which close to determine a given link type. Thus the problem of classifying link types is equivalent to the algebraic problem of classifying Markov classes [ $\beta$ ] in $B_{\infty}$.

In view of Markov's first move ((i) above) a necessary condition for a function with domain $B_{\infty}$ to be invariant on Markov classes is that it be a class function on each group $B_{n}$. We now review how Markov class invariants have been constructed out of class functions on representations of the braid groups. 


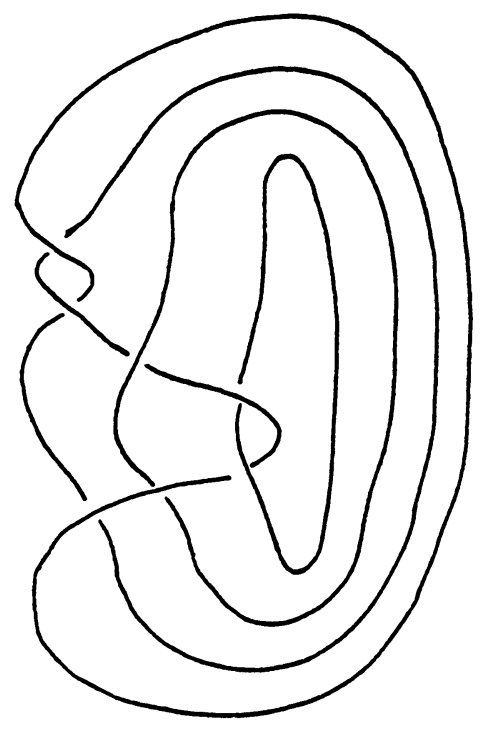

FIGURE 3

The Hecke algebra $\mathscr{H}_{n}(l, m)$ of the symmetric group is a 2-parameter family of algebras, with parameters $l, m \in \mathbf{C}$, and with $n \in \mathbf{N}$ a fixed natural number. For our purposes the most convenient definition of $\mathscr{H}_{n}(l, m)$ will be by generators and relations. Our generators will be denoted $\tilde{g}_{1}, \ldots, \tilde{g}_{n-1}$.

Notation. Instead of writing the relation $\tilde{g}_{i} \tilde{g}_{j}=\tilde{g}_{j} \tilde{g}_{i},|i-j| \geq 2, \ldots$, we will write $(1)_{\tilde{g}}$, and similarly $(2)_{\hat{g}}$ for relation (2) in the variables $\tilde{g}_{1}, \ldots, \tilde{g}_{n-1}$. With this convention, $\mathscr{H}_{n}(l, m)$ has defining relations $(1)_{\tilde{g}},(2)_{\tilde{g}}$ and

$$
l \tilde{g}_{i}+l^{-1} \tilde{g}_{i}^{-1}=m \text {. }
$$

The algebra $\mathscr{H}_{n}=\mathscr{H}_{m}(l, m)$ has been studied by Ocneanu [O] with a full account of the relevant features of his work given by Jones in [J,4]. The facts which we will need here are summarized by

Proposition 1.1 [J,4]. The algebras $\left\{\mathscr{H}_{n} ; n \in \mathbf{N}\right\}$ are ordered by inclusion $\mathscr{H}_{1} \subset$ $\mathscr{H}_{2} \subset \mathscr{H}_{3} \subset \ldots$, with $\mathscr{H}_{n}$ the subalgebra of $\mathscr{H}_{n+1}$ generated by $\tilde{g}_{1}, \ldots, \tilde{g}_{n-1}$. The algebra $\mathscr{H}_{n}$ has dimension $n$ !. Each $\mathscr{H}_{n}$ supports a trace function $\tau: \mathscr{H}_{n} \rightarrow$ $\mathrm{C}$, which is characterized uniquely by the conditions:

$$
\begin{gathered}
\tau(a+b)=\tau(a)+\tau(b) ; \\
\tau(a b)=\tau(b a) ; \\
\tau(1)=1 ; \\
\tau\left(w \tilde{g}_{n}\right)=\left(m^{-1}\left(l+l^{-1}\right)\right)^{-1} \tau(w) \quad \text { whenever } w \in \mathscr{H}_{n} \subset \mathscr{H}_{n+1} .
\end{gathered}
$$



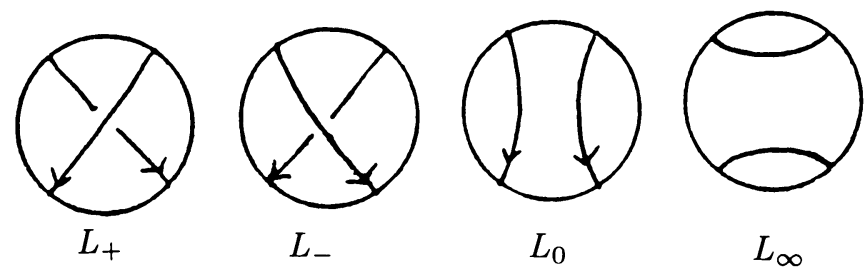

FIgURE 4

Note that (3), (4), (6) and (7) imply that:

$$
\tau\left(\tilde{g}_{i}\right)=\tau\left(\tilde{g}_{i}^{-1}\right)=\left(m^{-1}\left(l+l^{-1}\right)\right)^{-1}, \quad 1 \leq i \leq n-1 .
$$

The more common parametrization of $\mathscr{H}_{n}$ may be recovered by introducing new generators $g_{i}^{*}=t^{-1 / 2} \tilde{g}_{i}$ where $t$ is a new parameter, with $l=i t^{-1}$, $m=i\left(t^{1 / 2}-t^{-1 / 2}\right)$. Then (3) becomes

$$
\left(g_{i}^{*}\right)^{2}=(t-1) g_{i}^{*}+t
$$

This gives a 1-parameter version of the algebra $\mathscr{H}_{n}(l, m)$ which we will denote by the symbol $\mathscr{H}_{n}(t)$. A second parameter $z$ can then be introduced via the trace, if one replaces (7) by

$$
\tau\left(w g_{n}^{*}\right)=z \tau(w) \quad \text { whenever } w \in \mathscr{H}_{n}(t) \subset \mathscr{H}_{n+1}(t) .
$$

Equations $(3)^{*},(4),(6)$ and $(7)^{*}$ imply:

$$
\tau\left(g_{i}^{*}\right)=z, \quad \tau\left(g_{i}^{*-1}\right)=t^{-1} z+t^{-1}-1
$$

which is less convenient than (8) because of Markov's second move. This explains our choice of the generators $\tilde{g}_{i}$.

Since the trace is a class invariant in $\phi\left(B_{n}\right)$, and since (by (7) and (8)) it behaves nicely under the mapping $B_{n} \leftrightarrow B_{n+1}$ defined by Markov's second move ((ii) above) one sees immediately that the function

$$
P_{L}(l, m)=\left(m^{-1}\left(l+l^{-1}\right)\right)^{n-1} \tau(\phi(\beta))
$$

is invariant on the Markov class of each $\beta \in B_{\infty}$. It is the six-author polynomial of [FYHLMO].

Since the unknot is the closure of the 1 -braid $1 \in B_{1}$, one sees from (9) that for an unknotted circle:

$$
P_{\text {unknot }}(l, m)=1 \text {. }
$$

Let $L_{+}, L_{-}, L_{0}$ be links which are defined as the closures of $n$-braids $\beta_{+}$, $\beta_{-}, \beta_{0}$, where the defining braids are products of the elementary braids which are identical except for a single letter, with $\beta_{+}=\alpha \sigma_{i}, \beta_{-}=\alpha \sigma_{i}^{-1}, \beta_{0}=\alpha$, $\alpha \in B_{n}, 1 \leq i \leq n-1$. This means that $L_{+}, L_{-}, L_{0}$ have link diagrams which are identical everywhere except in a small disc, where they differ in the manner indicated in the first three pictures in Figure 4. It follows immediately from 
formula (3) and the linearity of the trace function that their polynomials are related by the crossing change formula

$$
l P_{L_{+}}(l, m)+l^{-1} P_{L_{-}}(l, m)=m P_{L_{0}}(l, m)
$$

This is Proposition 6.2 of $[\mathrm{J}, 4]$.

The key idea in the papers of Lickorish and Millett [L-M] and Hoste [H] was to use (10) and (11) as the basis of a definition of $P_{L}(l, m)$, thereby bypassing algebras, traces and all of the attendent machinery and focusing on the combinatorics of link diagrams. Their proof that $P_{L}(l, m)$ is a well-defined link invariant is equivalent to Jones' proof of Proposition 1.1. Our idea in the next section will be to reverse the procedure, using combinatorics to motivate a way to define a new algebra.

\section{The DEFINITION OF $\mathscr{C}_{n}(l, m)$}

For each natural number $n$ we define a 2-parameter family of algebras $\mathscr{C}_{n}(l, m)$ with generators $G_{1}, \ldots, G_{n-1}$ and relations $(1)_{G},(2)_{G}$, and others which involve elements $E_{1}, \ldots, E_{n-1}$ defined by

$$
G_{i}+G_{i}^{-1}=m\left(1+E_{i}\right)
$$

The additional relations are:

$$
\begin{aligned}
& E_{i} E_{i \pm 1} E_{i}=E_{i}, \\
& G_{i \pm 1} G_{i} E_{i \pm 1}=E_{i} G_{i \pm 1} G_{i}=E_{i} E_{i \pm 1}, \\
& G_{i \pm 1} E_{i} G_{i \pm 1}=G_{i}^{-1} E_{i \pm 1} G_{i}^{-1}, \\
& G_{i \pm 1} E_{i} E_{i \pm 1}=G_{i}^{-1} E_{i \pm 1}, \\
& E_{i \pm 1} E_{i} G_{i \pm 1}=E_{i \pm 1} G_{i}^{-1}, \\
& G_{i} E_{i}=E_{i} G_{i}=l^{-1} E_{i}, \\
& E_{i} G_{i \pm 1} E_{i}=l E_{i} .
\end{aligned}
$$

These imply the further relations:

$$
\begin{aligned}
& E_{i} E_{j}=E_{j} E_{i} \text { if }|i-j| \geq 2, \\
& E_{i}^{2}=\left(m^{-1}\left(l+l^{-1}\right)-1\right) E_{i}, \\
& G_{i}^{2}=m\left(G_{i}+l^{-1} E_{i}\right)-1 .
\end{aligned}
$$

In every case the indices are chosen from $1 \leq i, j \leq n-1$ to be all for which the relation in question makes sense.

Remark. Our choice of $l$ and $m$ as parameters suggests (as we will prove in $\S 3)$ that $\mathscr{H}_{n}(l, m)$ is a quotient of $\mathscr{C}_{n}(l, m)$. The homomorphism $\phi: B_{n} \rightarrow$ $\mathscr{H}_{n}(l, m)$ defined earlier will be seen to factor through $\mathscr{C}_{n}(l, m)$, via $\sigma_{i} \rightarrow$ $l^{-1} G_{i} \rightarrow \tilde{g}_{i}$. 
The rest of this section will be devoted to explaining our motivation in writing down these particular defining relations for $\mathscr{C}_{n}(l, m)$, using ideas from [K,1]. The reader who has not studied that paper may wish to omit the rest of this section on a first reading. The underlying idea is that we want to define our algebra in such a way as to force it to admit a trace, with the Kauffman polynomial a renormalization of that trace.

Recall that in $\S 1$ we showed that $P_{L}(l, m)$ satisfied the crossing-change formula (11). A similar situation exists for $K_{L}(l, m)$, but it requires a digression. Let $L$ be an oriented link which is defined by a link diagram $D$, i.e. a regular planar projection. Let $\varepsilon$ be the algebraic crossing number of the diagram, where crossings are counted as being positive (or negative) to correspond to the first (or second) picture in Figure 4. Kauffman defines in [K,1] a precurser of the polynomial $K_{L}(l, m)$, which he calls the " $L$-polynomial". We denote it by $\widehat{K}_{D}(l, m)$, defining it by:

$$
\widehat{K}_{D}(l, m)=l^{\varepsilon} K_{L}(l, m) .
$$

Note that $\widehat{K}_{D}(l, m)$ cannot be a link type invariant if $K_{L}(l, m)$ is, because the addition of a trivial loop in a link diagram, for example using Markov's second move, must leave $K_{L}(l, m)$ invariant but must then change $\widehat{K}_{D}(l, m)$. In fact, it must be true that:

If $D$ and $D^{\prime}$ are diagrams which are identical except for the addition of a trivial loop in $D^{\prime}$, which increases the algebraic crossing number, then $\widehat{K}_{D^{\prime}}(l, m)=l \widehat{K}_{D}(l, m)$.

Continuing, let $D_{+}, D_{-}, D_{0}, D_{\infty}$ be 4 link diagrams which are identical except inside a small disc, where they differ in the manner indicated in the four pictures in Figure 4. The $\widehat{K}_{D}$-polynomials $\widehat{K}_{D_{+}}, \widehat{K}_{D_{-}}, \widehat{K}_{D_{0}}, \widehat{K}_{D_{\infty}}$ which are associated to these diagrams are related by a crossing change formula, vis:

$$
\widehat{K}_{D_{+}}(l, m)+\widehat{K}_{D_{-}}(l, m)=m\left(\widehat{K}_{D_{0}}(l, m)+\widehat{K}_{D_{\infty}}(l, m)\right) .
$$

Two other properties of $\widehat{K}_{D}(l, m)$ are:

(23.4) If $D$ is a planar circle, then $\widehat{K}_{D}(l, m)=1$;

(23.5) If $D, D^{\prime}$ are diagrams which are related by regular isotopy in the plane (see $[\mathrm{K}, 1])$, then $\widehat{K}_{D}(l, m)=\widehat{K}_{D^{\prime}}(l, m)$.

The 5 axioms (23.2)-(23.5) suffice to determine $\widehat{K}_{D}(l, m)$ on all diagrams, and (adding (23.1)) to define $K_{L}(l, m)$ on all links. It is proved in [K,1] that $K_{L}(l, m)$ is well defined and a link type invariant, and that $\widehat{K}_{D}(l, m)$ is well defined on diagrams, and is an invariant of equivalence classes of diagrams, under "regular isotopy" of diagrams in the plane (see [K,1] for the definition of regular isotopy).

Our idea is as follows. Let $\beta=\sigma_{\mu_{1}}^{\varepsilon_{1}} \cdots \sigma_{\mu_{r}}^{\varepsilon_{r}}$ be a braid which closes to an oriented link $L=L_{\beta}$. The braid determines a diagram $D=D_{\beta}$ for the link 
$L$, and associated to this diagram is the polynomial $\widehat{K}_{D}(l, m)$. Eventually, we will define the trace (see $\S 3$ ) so that

$$
\operatorname{trace}\left(G_{\mu_{1}}^{\varepsilon_{1}} \cdots G_{\mu_{r}}^{\varepsilon_{r}}\right)=\left(m^{-1}\left(l+l^{-1}\right)-1\right)^{1-n} \widehat{K}_{D}(l, m) .
$$

Since $\widehat{K}_{D}(l, m)$ is well defined on diagrams, the trace will be seen to be well defined on monomials in the algebra. Then, if $g_{i}=l^{-1} G_{i}, 1 \leq i \leq n-1$, and if $\varepsilon=\varepsilon_{1}+\varepsilon_{2}+\cdots+\varepsilon_{n-1}$, this is equivalent to

$$
\operatorname{trace}\left(g_{\mu_{1}}^{\varepsilon_{1}} \cdots g_{\mu_{r}}^{\varepsilon_{r}}\right)=\left(m^{-1}\left(l+l^{-1}\right)-1\right)^{1-n} K_{L}(l, m),
$$

or (cf equation (9) above):

$$
K_{L}(l, m)=\left(m^{-1}\left(l+l^{-1}\right)-1\right)^{n-1} \operatorname{trace}\left(g_{\mu_{1}}^{\varepsilon_{1}} \cdots g_{\mu_{r}}^{\varepsilon_{r}}\right) .
$$

We now return to our algebra $\mathscr{C}_{n}(l, m)$, and to our attempts to motivate the relations in $\mathscr{C}_{n}(l, m)$. Recall from the discussion in $\S 1$ that if $L_{+}, L_{-}, L_{0}$ are as defined there, and if $L_{+}$is the closure of a braid $\beta_{+} \in B_{n}$, then it must be true that $\beta_{+}=\alpha \sigma_{i}, \beta_{-}=\alpha \sigma_{i}^{-1}, \beta_{0}=\alpha$, for some $\alpha \in B_{n}$, up to cyclic permutation of the defining braid. There seems to be no such braid interpretations for $L_{\infty}$, indeed $L_{\infty}$ cannot be defined by a braid in the usual sense.

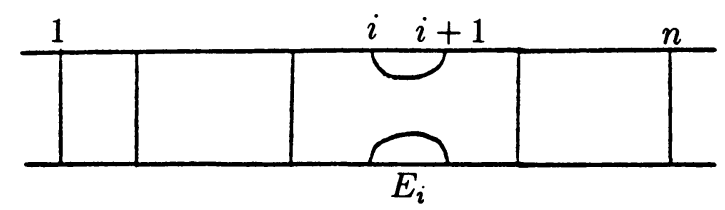

Figure 5

We introduce $(n-1)$ new elements $E_{1}, \ldots, E_{n-1}$, using (12) as our definition of $E_{i}$. Clearly $E_{i}$ ought to have come from a braid-like object in a "braid monoid", namely the embedding on $I^{(n)}=\coprod_{j=1}^{n} I_{j}$ in $\mathrm{C} \times I$ which goes with the picture in Figure 5, and it will be helpful to think of it in this way [Bo, Y, $\mathrm{K}, 2$ and $\mathrm{K}, 3]$.

There is no difficulty in composing such generalized braids with one another and with ordinary braids by concatenation. This allows us to interpret relations such as $E_{i} E_{i+1} E_{i}=E_{i}$ (relation (13)) and $G_{i+1} G_{i} E_{i+1} \approx E_{i} E_{i+1}$ (relation (14)) by pictures (Figure 6). Note that relations (13), (14), (15), (16), (17) are all associated to pictures which involve only regular isotopy. On the other hand, relations (18) and (19) require something more, because on the passage from the left side of (18) or (19) to the right we must delete a trivial loop, and axiom (23.2) asserts that this must be accounted for by the addition of a multiplicative 

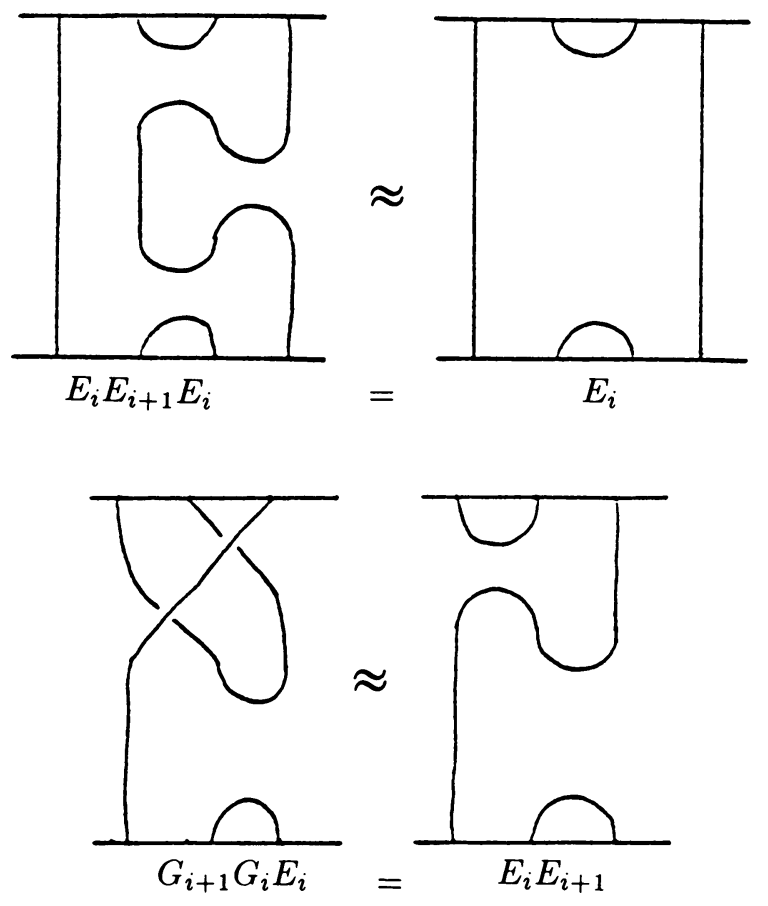

FIGURE 6

factor $l^{ \pm 1}$. See Figure 7. The remaining relations (20)-(22) are consequences of the others. Relation (20) follows from (1) ${ }_{G}$ and the definition of $E_{i}$ in (12). To obtain (21), multiply each term in (12) by $E_{i}$ and solve for $E_{i}^{2}$, using (18). To obtain (22), multiply each term in (12) by $G_{i}$ and solve for $G_{i}^{2}$, using (18). This accounts for all of the relations in $\mathscr{C}_{n}(l, m)$, and explains how we were led to our definition. In the next section we will study the algebra.

\section{Properties of $\mathscr{C}_{n}(l, m)$}

Lemma 3.1. $\mathscr{C}_{n}(l, m)$ is finite dimensional. Its dimension is bounded below by $n !$. Each element of $\mathscr{C}_{n}(l, m)$ can be written as a linear combination of elements of the form $w_{1} \gamma w_{2}$ with $\gamma \in\left\{G_{n-1}, E_{n-1}, 1\right\}$ and $w_{1}, w_{2}$ monomials in $1, G_{1}, E_{1}, \ldots, G_{n-2}, E_{n-2}$.

Proof. The mapping $\psi: \mathscr{C}_{n}(l, m) \rightarrow \mathscr{H}_{n}(l, m)$ defined by $G_{i} \rightarrow l g_{i}, E_{i} \rightarrow 0$ is a homomorphism, so $\operatorname{dim} \mathscr{C}_{n}(l, m)>\operatorname{dim} \mathscr{H}_{n}(l, m)=n !$.

To prove that $\operatorname{dim} \mathscr{C}_{n}(l, m)<\infty$, it suffices to prove that each $w \in \mathscr{C}_{n}=$ $\mathscr{C}_{n}(l, m)$ is a sum of monomials of the form $w^{\prime} \gamma w^{\prime \prime}$ where $w^{\prime}, w^{\prime \prime}$ belong to the subalgebra $\tilde{\mathscr{C}}_{n-1}$ of $C_{n}$ generated by $1, G_{1}, \ldots, G_{n-1}$ and where $\gamma=G_{n-1}$ or $G_{n-1}^{-1}$ or $E_{n-1}$ or 1 . The statement of the lemma then follows, because we can use (12) to eliminate $G_{1}^{-1}, \ldots, G_{n-1}^{-1}$.

If $n=2$ the assertion is trivially true, so assume $n>2$. Let $w$ be a monomial in $\mathscr{C}_{n}$. Then $w=w_{0} \gamma_{0} w_{1} \gamma_{1} \cdots w_{r} \gamma_{r}$, where each $w_{j} \in \tilde{\mathscr{C}}_{n-1}$ and 
each $\gamma_{j}=G_{n-1}, E_{n-1}$ or 1 . If $r=0$ we are done, so assume $r \geq 1$. Then $w=w_{0} \gamma_{0} w_{1} \gamma_{1} z$, where $z=w_{2} \gamma_{2} \cdots w_{r} \gamma_{r}$. Since $w_{1} \in \tilde{\mathscr{C}}_{n-1}$, induction on $r$ yields $w_{1}=v_{0} \alpha v_{1}$, where $v_{0}, v_{1} \in \widetilde{C}_{n-2}$ and $\alpha=G_{n-2}$ or $E_{n-2}$ or 1 . Then $v_{0}, v_{1}$ commute with $\gamma_{0}, \gamma_{1}$ by $(1)_{G}$ so $w=\left(w_{0} v_{0}\right)\left(\gamma_{0} \alpha \gamma_{1}\right)\left(v_{1} z\right)$, and it suffices to prove that the product $\gamma_{0} \alpha \gamma_{1}$ is a sum of monomials which involve $G_{n-1}$ or $E_{n-1}$ once, for all $\gamma_{0}, \gamma_{1} \in\left\{G_{n-1}, E_{n-1}\right\}$ and for $\alpha \in\left\{1, G_{n-2}, E_{n-2}\right\}$.

If $\alpha=1$, then relations (18), (21), (22) suffice to reduce each of these as claimed. Assume $\alpha \neq 1$. Then

$$
\begin{aligned}
& \gamma_{0} \alpha \gamma_{1} \in\left\{G_{n-1} G_{n-2} G_{n-1},\right. G_{n-1} G_{n-2} E_{n-1}, \\
& G_{n-1} E_{n-2} G_{n-1}, G_{n-1} E_{n-2} E_{n-1}, E_{n-1} G_{n-2} G_{n-1}, \\
&\left.\quad E_{n-1} G_{n-2} E_{n-1}, E_{n-1} E_{n-2} G_{n-1}, E_{n-1} E_{n-2} E_{n-1}\right\} .
\end{aligned}
$$

Using relations $(2)_{G},(12),(13),(14),(15),(16),(17)$, and (19) each of these can be reduced to a sum of monomials, each of which contains $E_{n-1}$ or $G_{n-1}$ once. Induction on $r$ completes the proof.

Remark. See Theorem 3.7 for a precise formula for $\operatorname{dim} \mathscr{C}_{n}(l, m)$.

We now show that our algebras support a trace function. The trace is most conveniently defined if we rescale the generators, so let $g_{i}=l^{-1} G_{i}, 1 \leq i \leq$ $n-1$.

Theorem 3.2. Each $\mathscr{C}_{n}(l, m)$ supports a trace function $\tau^{\prime}: \mathscr{C}_{n} \rightarrow \mathrm{C}$ which is characterized by the properties:

$$
\tau^{\prime}(a+b)=\tau^{\prime}(a)+\tau^{\prime}(b)
$$

$$
\tau^{\prime}(a b)=\tau^{\prime}(b a)
$$

$$
\tau^{\prime}(1)=1 \text { where } 1 \in \mathscr{C}_{n} \text { for any } n \in N,
$$

$$
\begin{aligned}
& \tau^{\prime}\left(w g_{n}\right)=\tau^{\prime}\left(w g_{n}^{-1}\right)=\tau^{\prime}\left(w E_{n}\right)=z \tau(w) \text { if } w \in \text { subalgebra of } \\
& \mathscr{C}_{n} \text { generated by } g_{1}, \ldots, g_{n-1}, \text { where } z^{-1}=m^{-1}\left(l+l^{-1}\right)-1 .
\end{aligned}
$$

Proof. There is an algebra homomorphism $\Delta: \mathbf{C} B_{n} \rightarrow \mathscr{C}_{n}=\mathscr{C}_{n}(l, m)$ which sends $\sigma_{i}$ to $g_{i}$. For any monomial $w=g_{\mu_{1}}^{\varepsilon_{1}} g_{\mu_{2}}^{\varepsilon_{2}} \cdots g_{\mu_{r}}^{\varepsilon_{r}} \in \mathscr{C}_{n}$, let $W=\sigma_{\mu_{1}}^{\varepsilon_{1}} \sigma_{\mu_{2}}^{\varepsilon_{2}} \cdots$ $\sigma_{\mu_{r}}^{\varepsilon_{r}} \in \Delta^{-1}(w)$. The braid $W$ determines a closed braid $\widehat{W}$ and so a link which we also refer to as $\widehat{W}$, with Kauffman polynomial $K(\widehat{W})$. Let

$$
\tau^{\prime}(w)=z^{n-1} K(\widehat{W}), \quad \text { where } z^{-1}=m^{-1}\left(l+l^{-1}\right)-1,
$$

and extend linearly to all of $\mathscr{C}_{n}(l, m)$.

For later use, note that by $(23.1)$ the link $\widehat{W}$ has diagram polynomial $\widehat{K}(\widehat{W})$ $=l^{\varepsilon_{1}+\varepsilon_{2}+\cdots+\varepsilon_{r}} K(\widehat{W})$.

Assuming for the moment that $\tau^{\prime}$ is well defined, independently of the choice of $W \in \Delta^{-1}(w)$, we note that $\tau^{\prime}$ satisfies properties $(4)^{\prime}-(7)^{\prime}$ : Property $(4)^{\prime}$ is 
satisfied by definition. Property $(5)^{\prime}$ holds because the closed braid $\widehat{W}$ depends only on the cyclic word $W$. Property (6) follows from the fact that $1 \in \mathscr{C}_{n}$ lifts to $1 \in B_{n}$, and $\hat{1}$ is the unlink of $n$-components, with Kauffman polynomial $z^{1-n}$. Thus $\tau^{\prime}(1)=z^{n-1} z^{1-n}=1$. Property $\left(7^{\prime}\right)$ follows from the fact that the links defined by closing the braids $W \in B_{n}$ and $W \sigma_{n}^{ \pm 1} \in B_{n+1}$ are equivalent, and so have the same Kauffman polynomial. It is immediate that these four properties determine $\tau^{\prime}$.

It remains to prove that $\tau^{\prime}$ is well defined. For this it suffices to show that $K$ takes the value 0 on the kernel of $\Delta$. Recall that $\mathbf{C} B_{n}$ is generated by $\sigma_{1}, \ldots, \sigma_{n-1}$, with defining relations $(1)_{\sigma}$ and $(2)_{\sigma}$, while $\mathscr{C}_{n}$ is generated by $G_{1}, \ldots, G_{n-1}$, with defining relations (12)-(19). Set $\xi_{1}=m^{-1}\left(l \sigma_{i}+l^{-1} \sigma_{i}^{-1}\right)-$ $1 \in B_{n}$. Then $\Delta\left(\xi_{i}\right)=E_{i}$. Lift $G_{i}$ to $l \sigma_{i}$. Then $\Delta\left(\sigma_{i}\right)=l^{-1} G_{i}$.

Each of the relations in (13)-(19) lifts to an element in the kernel of $\Delta$, say $R_{j} \quad(j=1, \ldots, q)$, and the kernel of $\Delta$ is the smallest two-sided ideal in $\mathrm{C} B_{n}$ spanned by the elements $R_{1}, \ldots, R_{q}$. Each $R_{j}$ is a linear combination of monomials in $C B_{n}$. For example, one of the relations in (18) yields:

$$
R_{1}=l^{2} \sigma_{i} \xi_{i}-\xi_{i} \text {. }
$$

If we use the symbol $\widehat{W} R_{j}$ for the sum of the closed braids determined by left-multiplying $R_{j}$ by an arbitrary monomial $W \in \mathbf{C} B_{n}$, we must prove that $K\left(\widehat{W} R_{j}\right)=0$. Note that we need only use left multiplication because closed braids belong to cyclic words in $B_{n}$.

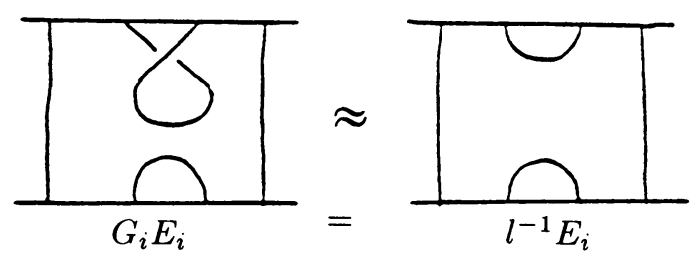

FIGURE 7

We do the calculation in the case of $R_{1}$, defined above. Recall that $R_{1}$ came from the relation (18), which was motivated by the pictures in Figure 7. We will use Figure 7 in a roundabout way, to be described. First, if we replace $\xi_{1}$ in $R_{1}$ by its expression as a sum of elementary braids $\sigma_{i}$ and $\sigma_{i}^{-1}$ we obtain:

$$
m R_{1}=l^{2}\left[l \sigma_{i}^{2}+l^{-1}-m \sigma_{i}\right]-\left[l \sigma_{i}+l^{-1} \sigma_{i}^{-1}-m\right] .
$$

Therefore, we must prove that

$l\left[l^{2} K\left(\hat{W} \sigma_{i}^{2}\right)+K(\widehat{W})-m l K\left(\widehat{W} \sigma_{i}\right)\right]-\left[l K\left(\widehat{W} \sigma_{i}\right)+l^{-1} K\left(\widehat{W} \sigma_{i}^{-1}\right)-m K(\widehat{W})\right]=0$.

Equivalently, via (23.1), we must prove that:

$$
l\left[\widehat{K}\left(\widehat{W} \sigma_{i}^{2}\right)+\widehat{K}(\widehat{W})-m \widehat{K}\left(\widehat{W} \sigma_{i}\right)\right]-\left[\widehat{K}\left(\widehat{W} \sigma_{i}\right)+\widehat{K}\left(\widehat{W} \sigma_{i}^{-1}\right)-m \widehat{K}(\widehat{W})\right]=0 .
$$


To do so, we first turn to Figure 4 , and recall the crossing-change formula (23.3) which relates the diagram polynomials of four links $L_{+}, L_{-}, L_{0}, L_{\infty}$ which are defined by diagrams which differ only in a small region, where the difference is as specified in Figure 4. Note that the closed braid diagrams for $\widehat{W} \sigma_{i}^{2}, \widehat{W}, \widehat{W} \sigma_{i}$ make up one such triplet $L_{+}, L_{-}, L_{0}$ and that $\widehat{W} \sigma_{i}, \widehat{W} \sigma_{i}^{-1}, \widehat{W}$ make up another $L_{+}^{\prime}, L_{-}^{\prime}, L_{0}^{\prime}$. Therefore if we bring the links $L_{\infty}$ and $L_{\infty}^{\prime}$ (with obvious notation) into the picture, we must show that

$$
l \widehat{K}\left(L_{\infty}\right)-\widehat{K}\left(L_{\infty}^{\prime}\right)=0
$$

where our links $L_{\infty}$ and $L_{\infty}^{\prime}$ are defined by diagrams which are identical everywhere except inside a region where they differ in the manner indicated in Figure 7, the picture on the left corresponding to $L_{\infty}$ and that on the right to $L_{\infty}^{\prime}$. Since Kauffman's axiom (23.2) asserts that $\widehat{K}\left(L_{\infty}^{\prime}\right)=l \widehat{K}\left(L_{\infty}\right)$ as required, and the proof is complete for $R_{1}$.

The other relations can be checked in the same way as the previous example. Note that it is enough to show that $\tau^{\prime}\left(R_{j}\right)=0$. Indeed, if $W \in B_{n}$ and $R_{j}$ is one of our relators, the links corresponding to $\widehat{W} R_{j}$ are the same everywhere except in a small area in which they differ from each other in the same way as the ones corresponding to $\widehat{R}_{j}$. So we can show that $K\left(\widehat{W} R_{j}\right)=0$ using the same crossing change rules coming from the definition of Kauffman's polynomial as we use for showing $K\left(\widehat{R}_{j}\right)=0$. This implies $\tau^{\prime}\left(W R_{j}\right)=0$.

The fact that $\tau^{\prime}\left(R_{j}\right)=0$ follows essentially from the definition of our relations which were motivated by pictures coming from the definition of Kauffman's link invariant (see the end of $\S 2$ ).

We now go on to determine the algebraic structure of $\mathscr{C}_{n}(l, m)$. Our main tool will be a generalized version of Jones' basic construction in $[\mathrm{J}, 1]$. For this, we will first have to extend methods already developed in [J,1 and W]. Our main observation is that the positivity assumptions in those papers are not necessary for our purposes. The only assumption will be that our traces are nondegenerate. This is made precise below.

We will assume throughout this section that $A<B$ are finite dimensional algebras over a field $S$ of characteristic zero. Let $M_{k}(S)$ be the algebra of all $k \times k$ matrices with entries from $S$. Assume that $A=\bigoplus A^{(i)}, B=\bigoplus B^{(j)}$ with $A^{(i)} \cong M_{a_{i}}(S), B^{(j)} \cong M_{b_{l}}(S)$ with $a_{i}, b_{j} \in \mathbf{N}$. The vectors $\vec{a}=\left(a_{i}\right)$ and $\vec{b}=\left(b_{j}\right)$ are called the dimension vectors of $A$ resp. $B$.

Let $\operatorname{tr}$ be a nondegenerate trace on $B$, i.e.

$$
\operatorname{tr}(x y)=0 \quad \forall y \in B \quad \text { implies } x=0 .
$$

It is well known and easy to check that, in our case, this is equivalent to $\operatorname{tr}(p) \neq 0$ for every minimal idempotent $p \in B$. Let us also recall that if $\operatorname{tr}$ is nondegenerate, the map $B \ni b \mapsto \operatorname{tr}(b \cdot) \in B^{*}$, the dual of $B$, is an isomorphism between 
$B$ and $B^{*}$ (as usual, $\operatorname{tr}(b \cdot)$, denotes the map $x \mapsto \operatorname{tr}(b x)$ ), i.e. for any linear map $\varphi: B \rightarrow S$ there is a unique $b \in B$ such that $\varphi(x)=\operatorname{tr}(b x)$ for all $x \in B$.

Let us also assume that the restriction of $\operatorname{tr}$ onto $A$ is nondegenerate. Then, using the isomorphism above for $A$ and $A^{*}$, there exists for every $b \in B$ a necessarily unique $\varepsilon_{A}(b) \in A$ such that

$$
\left.\operatorname{tr}(b \cdot)\right|_{A}=\left.\operatorname{tr}\left(\varepsilon_{A}(b) \cdot\right)\right|_{A} .
$$

It can be checked easily that the map $B \ni b \rightarrow \varepsilon_{A}(b) \in A$ is linear and that

$$
\begin{gathered}
\operatorname{tr}(b)=\operatorname{tr}\left(\varepsilon_{A}(b)\right), \\
\varepsilon_{A}\left(a_{1} b a_{2}\right)=a_{1} \varepsilon_{A}(b) a_{2}, \quad a_{1}, a_{2} \in A, b \in B .
\end{gathered}
$$

We call $\varepsilon_{A}$ the trace preserving conditional expectation from $B$ to $A$. Let us represent $B$ via the left regular representation onto itself. To avoid confusion we write $B_{\xi}$ if we regard $B$ as the representation space and $b_{\xi}$ for an element of $B_{\xi}$. Let $\mathscr{L}\left(B_{\xi}\right)$ be the set of all linear maps on $B_{\xi}$. For any algebra $C$ on $B_{\xi}$ let

$$
C^{\prime}=\left\{x \in \mathscr{L}\left(B_{\xi}\right), x c=c x, \forall c \in C\right\} .
$$

It is well known (and can be checked by explicit matrix multiplications) that $C$ is isomorphic to a direct sum of full matrix algebras iff $C^{\prime}$ is isomorphic to a direct sum of full matrix algebras. If $B$ (and $B_{\xi}$ ) is finite dimensional it follows that $\left(B^{\prime}\right)^{\prime}=B$ (see for instance [La, XVIII, 3, Theorem 1]).

By our assumptions on the structure of $B$, we can regard $B$ as a concrete matrix algebra such that with $b \in B$, its transpose, denoted here by $b^{*}$, is in $B$. Moreover, we can choose this matrix representation such that with $a$ also $a^{*}$ is in $A \subset B$. Note that ${ }^{*}: B \rightarrow B, b \mapsto b^{*}$ is an involution with $(a b)^{*}=b^{*} a^{*}$. We can then define a map $J: B_{\xi} \rightarrow B_{\xi}$ by $J\left(b_{\xi}\right)=b_{\xi}^{*}$. A straightforward computation shows that $J B J \subseteq B^{\prime}$. On the other hand, if $b^{\prime} \in B^{\prime}$ such that $b^{\prime}\left(1_{\xi}\right)=b_{\xi}$, we have $b^{\prime}\left(a_{\xi}\right)=a b_{\xi}=\left(J b^{*} J\right)\left(a_{\xi}\right)$ for any $a \in B$. Hence $J B J=B^{\prime}$. As in $[\mathrm{J}, 1]$, we define $e_{A} \in L\left(B_{\xi}\right)$ by $e_{A}\left(b_{\xi}\right)=\left(\varepsilon_{A}(b)\right)_{\xi}$.

Lemma 3.3. With the above notation, we have

(i) $e_{A} x e_{A}=\varepsilon_{A}(x) e_{A}$ for $x \in B$.

(ii) Let $x \in B$. Then $x \in A$ iff $e_{A} x=x e_{A}$.

(iii) $A^{\prime}=\left\{B^{\prime} \cup\left\{e_{A}\right\}\right\}^{\prime \prime}$.

(iv) $J$ commutes with $e_{A}$.

Proof. See [J, (3.1.4)]. For (ii), replace the statement " $\xi$ is separating" by "the map $B \ni b \rightarrow b_{\xi} \in B_{\xi}$ is injective".

Obviously, a simple $B^{(j)}$ module is also an $A$ module. Let $g_{i j}$ be the number of simple $A^{(i)}$ modules in its decomposition into simple $A$ modules. The matrix $G=\left(g_{i j}\right)$ is called the inclusion matrix for $A \subset B$. If $A$ and $B$ have the same identity element, we have

$$
\vec{b}=G^{t} \vec{a} \text {. }
$$


Under these assumptions we can give a precise description of $\left\langle B, e_{A}\right\rangle$, the algebra generated by $B$ and $e_{A}$. As in [J,1], we call $\left\langle B, e_{A}\right\rangle$ the basic construction for $A \subset B$.

Proposition 3.4. (i) $\left\langle B, e_{A}\right\rangle \cong A^{\prime}$. In particular, $\left\langle B, e_{A}\right\rangle$ is isomorphic to a direct sum of full matrix algebras.

(ii) There exists a canonical 1-1 correspondence between the direct summands of $A$ and $\left\langle B, e_{A}\right\rangle$ such that if $q$ is a minimal idempotent of $A^{(i)}, e_{A} q$ is a minimal idempotent of $\left\langle B, e_{A}\right\rangle^{(i)}$.

(iii) The inclusion matrix for $B \subset\left\langle B, e_{A}\right\rangle$ is $G^{t}$.

(iv) Every element $x \in\left\langle B, e_{A}\right\rangle$ can be written in the form $x=\sum a_{i} e_{A} b_{i}+c$, where $a_{i}, b_{i} c \in B$.

Proof. (i) As $A^{*}=A$, it follows as in $[\mathrm{J}, 1,(3.1 .5)]$ that $\left\langle B, e_{A}\right\rangle=J A^{\prime} J$.

(ii)-(iv) follow from [J,1, (3.3.1)].

The following theorem is an extension of [W, Proposition (1.2)] to semisimple algebras.

Theorem 3.5. Let $A, B, \operatorname{tr}$ and $\varepsilon_{A}$ be as above. Assume that $B$ is contained in an algebra $C$ and there is an element $e \in C$ such that

(i) $e^{2}=e$,

(ii) exe $=\varepsilon_{A}(x) e=e \varepsilon_{A}(x)$ for all $x \in B$,

(iii) The map $a \in A \mapsto$ ae $\in\langle B, e\rangle$ is an injective homomorphism with $e 1=e$.

Then the 2-sided ideal $\langle e\rangle \subset\langle B, e\rangle$ generated by $e$ is isomorphic to $\left\langle B, e_{A}\right\rangle$ as an algebra. The quotient $\langle B, e\rangle /\langle e\rangle$ is isomorphic to a subalgebra of $B$ and splits as a direct summand in $\langle B, e\rangle$.

Proof. For convenience, let us not distinguish between $B \subset C$ and the isomorphic image of $B$ in the representation on $B_{\xi}$. Let us define $\varphi: B \cup\{e\} \rightarrow \mathscr{L}\left(B_{\xi}\right)$ by $b \mapsto b . \in \mathscr{L}\left(B_{\xi}\right)$ and $e \mapsto e_{A}$. We claim that $\varphi$ extends to a well-defined homomorphsim from $\langle B, e\rangle$ onto $\left\langle B, e_{A}\right\rangle$. The only nontrivial part is to show that $\varphi$ is well defined. Let $x=\sum a_{i} e b_{i}+c=0, a_{i}, b_{i}, c \in B$. (It follows from (i)-(iii) that this is the most general form of an element of $\langle B, e\rangle$; see also $[\mathrm{J}, 1]$.) Let $\tilde{y} . \in B$ and let $\tilde{x}=\sum a_{i} \varepsilon_{A}\left(b_{i} \tilde{y}\right)+c \tilde{y}$. Then $0=e y x e=\varepsilon_{A}(y \tilde{x}) e$ $\forall y . \in B$. By (iii), $\varepsilon_{A}(y \tilde{x})=0$ and therefore also $\operatorname{tr}(y \tilde{x})=0$ for all $y . \in B$. Hence $\tilde{x}=0$ and we obtain

$$
\varphi(x)(\tilde{y} \xi)=\tilde{x} \xi=0 .
$$

As $\tilde{y}$ was arbitrary, $\varphi(x)=0$.

On the other hand, let $x=\sum a_{i} e b_{i}+c . \in k e \varphi$. Then $0=\varphi(x)(\tilde{y} \xi)=$ $\left(\sum a_{i} \varepsilon_{A}\left(b_{i} \tilde{y}\right)+c \tilde{y}\right) \xi \quad \forall \tilde{y}=B$. Hence $\sum a_{i} \varepsilon_{A}\left(b_{i} \tilde{y}\right)+c \tilde{y}=0$ and in particular,

$$
x \tilde{y} e=0 \quad \forall \tilde{y} \in B \text {. }
$$


But then we also have

$$
\begin{aligned}
\operatorname{tr}\left(y\left(\sum a_{i} \varepsilon_{A}\left(b_{i} \tilde{y}\right)+c \tilde{y}\right)\right) & =\operatorname{tr}\left(\sum \varepsilon_{A}\left(y a_{i}\right) \varepsilon_{A}\left(b_{i} \tilde{y}\right)+y c \tilde{y}\right) \\
& \left.=\operatorname{tr}\left(\sum \varepsilon_{A}\left(y a_{i}\right) b_{i}+y c\right) \tilde{y}\right)=0
\end{aligned}
$$

for all $y, \tilde{y} . \in B$.

So, as tr is nondegenerate, we obtain $\sum \varepsilon_{A}\left(y a_{i}\right) b_{i}+y c=0$ and in particular $(* *)$

$$
\text { eyx }=0 \quad \forall y . \in B .
$$

Hence if $x \in \operatorname{ker} \varphi$ and $y \in B e B$, we have $x y=y x=0$ by $(*)$ and $(* *)$. In particular, if $z \in \operatorname{ker} \varphi \cap B e B, z$ is annihilated both by $B e B$ and $\operatorname{ker} \varphi$.

As $\varphi(B e B)=\left\langle B, e_{A}\right\rangle$ (see Proposition 3.4, (iv)), $B e B$ and $\operatorname{ker} \varphi$ generate $\langle B, e\rangle$. So $z=1 z=0$, as by (iii) $1 \in B$ is also the multiplicative identity in $\langle B, e\rangle$. It follows from this that $B e B \cong\left\langle B, e_{A}\right\rangle$, which is a direct sum of full matrix algebras.

By (i)-(iii), $\langle e\rangle \cong B e B$. Obviously, the quotient $\langle B, e\rangle /\langle e\rangle$ is already generated by the image of $B$, hence it is isomorphic to a subalgebra of the semisimple algebra $B$. It is well known that in this case

$$
\langle B, e\rangle \cong\langle e\rangle \oplus\langle B, e\rangle /\langle e\rangle \text {. }
$$

Lemma 3.6. Let $e_{n}=z E_{n}$, where $z=1 /\left(m^{-1}\left(l+l^{-1}\right)-1\right)$ and assume that $\tau^{\prime}$ is nondegenerate on $C_{n-1}$ and $C_{n}$. Then $e_{n}$ has properties (i)-(iii) of Theorem 3.5 for $C_{n-1} \subset C_{n}$ and $C_{n-1}$ is isomorphic to the subalgebra of $C_{n}$ generated by $G_{1}, \ldots, G_{n-2}, E_{1}, \ldots, E_{n-2}$.

Proof. Using Theorem 3.2, (7) and the fact that $\tau^{\prime}$ is nondegenerate we can show easily that $\mathscr{C}_{n-1} \ni x \mapsto x e_{n} \in \mathscr{C}_{n+1}$ is injective. From this follows the last statement of the lemma and property (iii). By Lemma 3.1, any element of $\mathscr{C}_{n}(l, m)$ can be written as a linear combination of elements of the form $a \chi b$ with $a, b \in \mathscr{C}_{n-1}(l, m)$ and $\chi \in\left\{1, G_{n-1}, E_{n-1}\right\}$.

By Theorem 3.2, (7) ${ }^{\prime}$ we obtain for $\chi$ as above and $c \in \mathscr{C}_{n-1}(l, m)$

$$
\tau^{\prime}((a \chi b) c)=\tau^{\prime}(\chi) \tau^{\prime}(a b c) \text {. }
$$

Hence $\varepsilon(a \chi b)=\tau^{\prime}(\chi) a b$ where

$$
\varepsilon: \mathscr{C}_{n}(l, m) \rightarrow \mathscr{C}_{n-1}(l, m)
$$

is the trace preserving conditional expectation. On the other hand

$$
\begin{aligned}
e_{n}(a \chi b) e_{n} & =z^{2} a E_{n} \chi E_{n} b= \begin{cases}z^{2} a E_{n} b & \text { if } \chi=E_{n-1}, \\
l z^{2} a E_{n} b & \text { if } \chi=G_{n-1},\end{cases} \\
& =\tau^{\prime}(\chi) a b e_{n}=\varepsilon(a \chi b) e_{n} .
\end{aligned}
$$

This shows property (ii). Property (i) follows from (21).

We can now determine the structure of $C_{n}$ as an algebra over an appropriate field. 
Theorem 3.7. Let us regard $C_{n+1}=C_{n+1}(l, m)$ as an algebra over $\mathbf{C}(l, \alpha)$ where $m=\alpha+\alpha^{-1}$. Then $C_{1} \cong \mathbf{C}(l, \alpha), C_{2} \cong \mathbf{C}(l, \alpha)^{3}$ and

$$
C_{n+1} \cong H_{n+1} \oplus\left\langle C_{n}, e_{C_{n-1}}\right\rangle \text {, }
$$

where $H_{n+1}=H_{n+1}\left(l, \alpha+\alpha^{-1}\right)$ and the second summand is Jones' basic construction for $C_{n-1} \subset C_{n}$, which is semisimple. In particular $C_{n+1}$ is semisimple, it has dimension $1 \cdot 3 \cdot 5 \cdots(2 n+1)$ and $\tau^{\prime}$, the trace as defined in Theorem 3.2 , is nondegenerate.

Proof. Clearly, $C_{1} \cong \mathbf{C}(l, \alpha)$. Let $z$ be the rational function in $l$ and $m=$ $\alpha+\alpha^{-1}$ as defined in the previous lemma and let

$$
p_{1}=z E_{1}, \quad p_{2}=\frac{G_{1}-\alpha}{\alpha^{-1}-\alpha}\left(1-p_{1}\right) \quad \text { and } \quad p_{3}=\frac{G_{1}-\alpha^{-1}}{\alpha-\alpha^{-1}}\left(1-p_{1}\right) .
$$

Then it follows from (12), (18) and (21) that $p_{i}^{2}=p_{i}, p_{i} p_{j}=0$ for $i, j=$ $1,2,3$, and $i \neq j$ and that $p_{1}+p_{2}+p_{3}=1$. Using property (7) $)^{\prime}$ of Theorem (3.2) one sees that $\tau^{\prime}\left(p_{i}\right) \neq 0$ for $i=1,2,3$ which shows the assumptions for $C_{2}$.

It has already been pointed out earlier that the additional relation $E_{i}=0$ reduces the generating relations of $C_{n}$ to the defining relations of the Hecke algebra $H_{n}$. We thus obtain a homomorphism $\psi$ from $C_{n}$ onto $H_{n}$. By definition of $\psi$, its kernel is equal to the ideal $I$ generated by the $E_{i}$ 's. By a theorem of Benson and Curtis (see [Lu, Theorem (3.1)])

$$
C_{n} / \operatorname{ker} \psi \cong H_{n} \cong \mathbf{C}(l, \alpha) S_{n},
$$

where $S_{n}$ denotes the symmetric group on $n$ letters.

Using relation (13) repeatedly, we obtain

$$
E_{i}=E_{i} E_{i+1} \cdots E_{n-1} E_{n} E_{n-1} \cdots E_{i+1} E_{i} .
$$

Hence $I$ coincides with the ideal $\left\langle E_{n}\right\rangle \subset C_{n+1}$ generated by $E_{n}$. Using relations (13), (16) and (17), we show as in Lemma 3.1 that $\left\langle E_{n}\right\rangle$ is the linear span of products of generators of the form $w_{1} E_{n} w_{2}$ with $w_{1}, w_{2} \in C_{n}$. It follows from this, Theorem 3.5 and Lemma 3.6 that

$$
\operatorname{ker} \psi=\left\langle E_{n}\right\rangle=C_{n} E_{n} C_{n} \cong\left\langle C_{n}, e_{C_{n-1}}\right\rangle .
$$

We shall show at the end of this section by a simple dimension argument that $\left\langle C_{n}, e_{C_{n-1}}\right\rangle=\left\langle C_{n}, e_{C_{n-1}}\right\rangle^{\prime \prime}$. So by Proposition (3.4), (i) ker $\psi$ is semisimple which is also true for the quotient $C_{n+1} / \operatorname{ker} \psi$ by $(*)$. Hence

$$
C_{n+1} \cong H_{n+1} \oplus C_{n} E_{n} C_{n} \text {. }
$$

It remains to show that $\tau^{\prime}$ is nondegenerate. Let $p$ be a minimal projection of $C_{n-1}$. Then $\tau^{\prime}\left(e_{n} p\right)=z^{2} \tau^{\prime}(p) \neq 0$ by induction assumption. So $\tau^{\prime}$ is nondegenerate on $C_{n} E_{n} C_{n}$ by Proposition (3.4), (ii) and the remark below (25). 
Note that if $l=1$ and $\alpha=i, z(1, i)=0$ and $\tau^{\prime}\left(p_{2}\right)(1, i)=\tau^{\prime}\left(p_{3}\right)(1, i)=$ $1 / 2$. By the induction assumption $\tau^{\prime}(p)(1, i)=0$ for any minimal projection in $C_{n-2} E_{n-2} C_{n-2}$ and $\tau^{\prime}(p)(1, i) \neq 0$ for any minimal projection in the $H_{n-1}$ summand of $C_{n-1}$. It follows as in the previous paragraph that $\tau^{\prime}(p)(1, i)=0$ for any minimal projection in $C_{n} E_{n} C_{n}$. So $\tau^{\prime}$ can be considered as a trace on $H_{n+1}$ having Markov property $(7)^{\prime}$. These traces are uniquely determined by $q=-\alpha^{2}$ and $\eta=\left(\operatorname{tr}\left(\tilde{g}_{i}\right)+1\right) /(1+q)$. For our special choice of values we obtain $q=1$ and $\eta=1 / 4$. But then it follows from Ocneanu's classification of positive traces on $H_{\infty}$ that $\tau^{\prime}(p)(1, i)>0$ for all minimal projections in $H_{n+1}$ (see [W, proof of Theorem (3.5), (i), $\alpha$ ]). Hence $\tau^{\prime}(p) \neq 0$ for any minimal projection $p$ in the $H_{n+1}$-summand of $C_{n+1}$. This shows that $\tau^{\prime}$ is nondegenerate on $C_{n+1}$.

We next describe how to use "Bratteli diagrams" to determine the structure of $C_{n}(l, \alpha)$. These diagrams allow one to compute the irreducible representations of these algebras by an iterative procedure. We will first assume that $\left\langle C_{n}, e_{C_{n-1}}\right\rangle=\left\langle C_{n}, e_{C_{n-1}}\right\rangle^{\prime \prime}$, where the right-hand side is semisimple by Proposition 3.4. We will then show by computing dimensions that the left-hand side cannot be a proper subalgebra of the right-hand side. An example will be given after we describe the basic setup. Related constructions are to be found in [ $\mathrm{J}, 2]$ (for the algebra we called $A_{n}(t)$ in the introduction) and in [W] (for $\mathscr{H}_{n}(t)$ ). See also [J,4], where the underlying construction for the group algebra $\mathbf{C} S_{n}$ of the symmetric group by "Young diagrams" is described in a particularly lucid manner.

The Bratteli diagram for $\mathscr{C}_{n}(l, m)$ is a graph which we now describe. See Figure 8. The vertices are arranged in horizontal rows, those in the $n$th row from the top being in 1-1 correspondence with the irreducible representations of $\mathscr{C}_{n}(l, m), n=1,2,3, \ldots$ A vertex in row $n$ is joined to one in row $n-1$ by $k$ edges if the restriction of the corresponding representation of $\mathscr{C}_{n}$ to $\mathscr{C}_{n-1}$, under the natural inclusion $\mathscr{C}_{n-1} \hookrightarrow \mathscr{C}_{n}$, contains that irreducible representation of $\mathscr{C}_{n-1} k$ times. The vertices are labeled by integers which denote the dimension; the dimension of a representation of $\mathscr{C}_{n}$ is the sum of the dimensions of all those representations of $\mathscr{C}_{n-1}$ which are joined to it by edges. Note that $\mathscr{C}_{n}=\mathscr{H}_{n}^{\prime} \oplus \mathscr{H}_{n}$ by Theorem 3.7 , where $\mathscr{H}_{n}^{\prime}$ is $\mathscr{C}_{n} E_{n} \mathscr{C}_{n}$. We now describe how to construct row $n$ of the diagram from row $n-1$, $n \geq 3$. One first reflects that part of the diagram which consists of the vertices in rows $n-2$ and $n-1$ and all edges joining them about the horizontal line corresponding to the representations of $\mathscr{C}_{n-1}$. One then inserts the dimension of the representations of $\mathscr{C}_{n-1}$, and assigns to each representation of $\mathscr{C}_{n}$ the sum of the dimensions of the representations lying above it. See Figure 8. This determines that part of $\mathscr{C}_{n}$ which we have called $\mathscr{H}_{n}^{\prime}$. One then augments it by adding more vertices, one for each irreducible representation of $\mathscr{H}_{n}$, using the conventions in [W] (or in [J,2]; see especially $\S 3$ of [J,2]). This makes sense 
because each $\mathscr{C}_{j}, j<n$, has already been constructed as $\mathscr{H}_{j}^{\prime} \oplus \mathscr{H}_{j}$, so that the Bratteli diagram for $\left\{\mathscr{H}_{n} ; n=1,2,3, \ldots\right\}$ is embedded as a subdiagram of that for $\left\{\mathscr{C}_{n} ; n=1,2,3, \ldots\right\}$. Finally, we note that the inductive construction begins with the unique 1-dimensional trivial representation of $\mathscr{C}_{1}$, and three 1-dimensional representations of $\mathscr{C}_{2}$, one belonging to $\mathscr{H}_{2}^{\prime}$ and the other two to $\mathscr{H}_{2}$.

Figure 8 illustrates the Bratteli diagram through $n=4$, and shows the construction of $\mathscr{B}_{5}^{\prime}$ from that of $\mathscr{C}_{4}$ and $\mathscr{C}_{3}$. Later, in $\S 6$, we will use this diagram to obtain explicit matrix representations of $B_{3}$ and $B_{4}$.

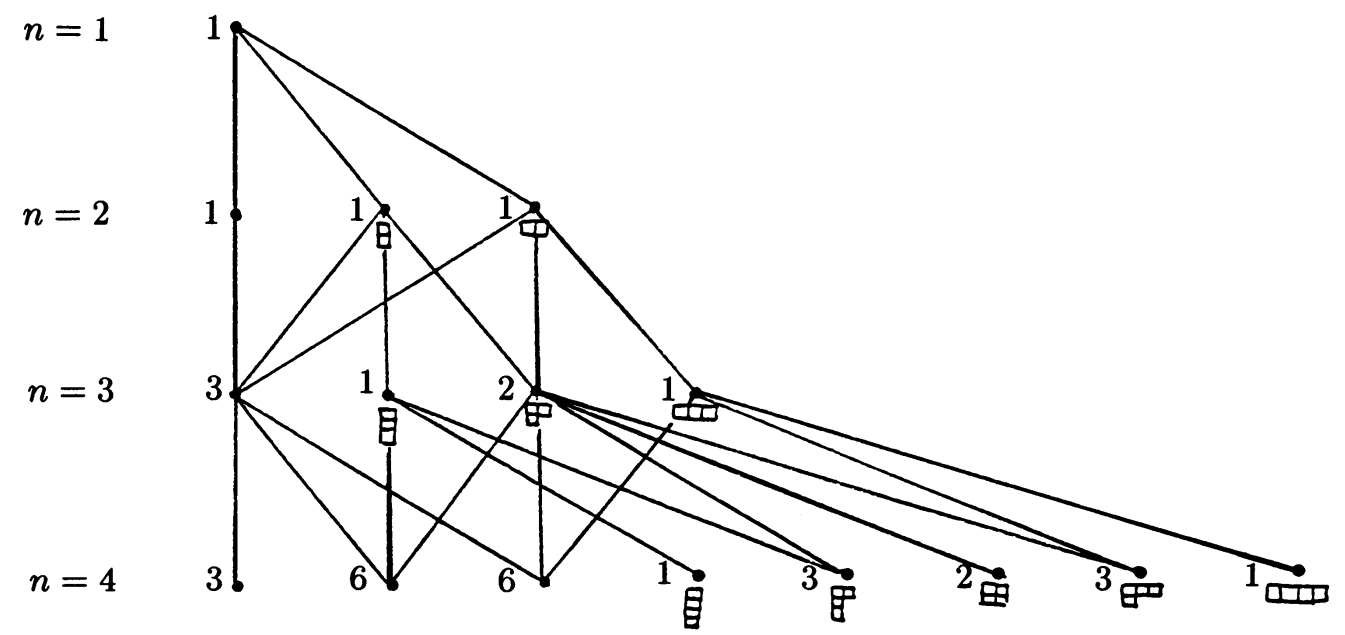

(a) The construction of $H_{n}^{\prime} \oplus H_{n}, 1 \leq n \leq 4$

$n=3$

$n=5$

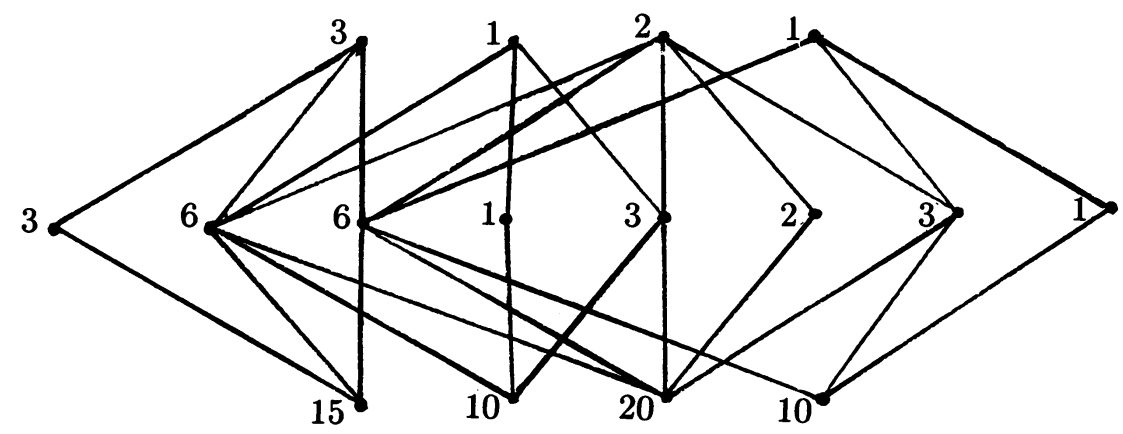

(b) The construction of $H_{n}^{\prime}, n=5$

Figure 8 
We now show the missing part in the proof of Theorem 3.7, namely that $\left\langle C_{n}, e_{C_{n-1}}\right\rangle=\left\langle C_{n}, e_{C_{n-1}}\right\rangle^{\prime \prime}$. It is a well-known combinatorial fact that the algebras constructed by this procedure (i.e. with $\left\langle C_{n}, e_{C_{n-1}}\right\rangle^{\prime \prime}$ instead of $\left.\left\langle C_{n}, e_{C_{n-1}}\right\rangle\right)$ have dimension $1 \cdot 3 \cdots(2 n+1)$ (see for instance [S, Lemma 8.7]). On the other hand, we shall see in $\S 5$ that for a special choice of (slightly modified) parameters $C_{n+1}(l, \alpha)$ specializes to Brauer's algebra $D_{n+1}(x)$ which has dimension $1 \cdot 3 \cdots(2 n+1)$. So also the dimension of $C_{n+1}(l, \alpha)$ has to be at least that number. This shows again by induction on $n$ that $\left\langle C_{n}, e_{C_{n-1}}\right\rangle=$ $\left\langle C_{n}, e_{C_{n-1}}\right\rangle^{\prime \prime}$.

\section{HOMOMORPHISMS ONTO $A_{n}(t)$}

Let $A_{n}(t)$ denote the one-parameter family of algebras studied by Jones in [J,2], with idempotent generators $e_{1}, \ldots, e_{n-1}$ and defining relations:

$$
\begin{gathered}
e_{i} e_{i \pm 1} e_{i}=\left(t^{1 / 2}+t^{-1 / 2}\right)^{-2} e_{i}, \\
e_{i}^{2}=e_{i}, \\
e_{i} e_{j}=e_{j} e_{i} \quad \text { if }|i-j| \geq 2 .
\end{gathered}
$$

Jones shows that $A_{n}(t)$ is semisimple, and that its irreducible representations are in 1-1 correspondence with those irreducible representations of $\mathscr{H}_{n}(l, m)$ which are associated to Young diagrams having one or two rows. Thus $A_{n}(t)$ is a homomorphic image of $\mathscr{H}_{n}(l, m)$. He also shows that there are homomorphisms from $B_{n}$ into $A_{n}(t)$ which map the elementary braid $\sigma_{i}$ to an appropriate linear combination of $e_{i}$ and 1 , which we will denote by $\hat{g}_{i}$ here.

We now show that there are two inequivalent homomorphisms $\rho$ and $\psi$ from $\mathscr{C}_{n}(l, m) \rightarrow A_{n}(t)$. Inequivalent means that there cannot be an isomorphism $i$ of $\mathscr{C}_{n}(l, m)$ with $\rho=\psi \circ i$.

(i) Define $\rho_{1}: \mathscr{C}_{n}(l, m) \rightarrow \mathscr{H}_{n}(l, m)$ by $\rho_{1}\left(E_{i}\right)=0, \rho_{1}\left(g_{i}\right)=\tilde{g}_{i}$, and $\rho_{2}: \mathscr{H}_{n}(l, m) \rightarrow A_{n}(t)$ by $\rho_{2}\left(\tilde{g}_{i}\right)=t^{1 / 2} \hat{g}_{i}$, and $(l, m)=\left(i t^{-1}, i\left(t^{1 / 2}-t^{-1 / 2}\right)\right)$. Let $\rho=\rho_{2} \circ \rho_{1}$. The homomorphism $\rho$ was studied in detail in [J,2]. Using the Bratteli diagram for $\mathscr{C}_{n}(l, m)$ which is described in Figure 8 above, the map $\rho_{1}$ may be realized by deleting those representations which belong to vertices which are not labeled by Young diagrams, while $\rho_{2}$ deletes those from among the remaining representations in which the Young diagrams have more than two rows.

(ii) It has been shown by Lickorish (see [Li]) that the Jones polynomial can be obtained from the Kauffman polynomial for the special choice of parameters $(l, m)=\left(t^{-3 / 4},-t^{1 / 4}-t^{-1 / 4}\right)$. Hence, by factoring over the ideal $I_{n}$ defined by

$$
I_{n}=\left\{a \in C_{n}\left(t^{-3 / 4},-t^{1 / 4}-t^{-1 / 4}\right), \tau^{\prime}(a b)=0\right.
$$

$$
\text { for all } \left.b \in C_{n}\left(t^{-3 / 4},-t^{1 / 4}-t^{-1 / 4}\right)\right\} \text {, }
$$


we obtain a well-defined surjective homomorphism

$$
\Psi: C_{n}\left(t^{-3 / 4},-t^{1 / 4}-t^{-1 / 4}\right) \mapsto C_{n}\left(t^{-3 / 4},-t^{1 / 4}-t^{-1 / 4}\right) / I_{n} \cong A_{n}(t) .
$$

This homomorphism can be described explicitly by (see also [J,2, §3])

$$
\Psi\left(G_{i}\right)=t^{-1 / 4}\left((1+t) e_{i}-1\right)
$$

which implies

$$
\Psi\left(E_{i}\right)=-\left(t^{1 / 2}+t^{-1 / 2}\right) e_{i} .
$$

The last equality shows that $\Psi$ does not factor through $H_{n}$. An explicit computation shows that $\tau^{\prime}$ annihilates the idempotent $p_{3}$ as defined in the proof of Theorem 3.7 for the special choice $\alpha=-t^{1 / 4}$ and $l=t^{-3 / 4}$. Hence the hypotheses of Theorem 3.5 are no longer satisfied. In particular, we can no longer construct Bratteli diagrams as in Theorem 3.7. Similar phenomena with Brauer's centralizer algebras (see next section and [W,2]) suggest that $C_{3}$ (and also higher $C_{n}$ 's) will no longer be semisimple for this special choice of $l$ and $m$. Thus the kernel of $\Psi$ sits in a complicated way in $C_{n}(l, m)$ completely different from our first kernel.

\section{BRAUER'S CENTRALIZER ALGEBRAS}

In $1937 \mathrm{R}$. Brauer introduced, in [Br], a family of algebras which we denote by $\left\{\mathscr{D}_{n}(x)\right\}$, where $n$ is a positive integer and $x$ a parameter or indeterminate. His algebras are ordered by inclusion, $\mathscr{D}_{1}(x) \subset \mathscr{D}_{2}(x) \subset \cdots$. We will show that our algebras are perturbations or deformations of $\mathscr{D}_{n}(x)$ in much the same way as the Hecke algebra $\mathscr{H}_{n}(t)$ of type $A_{n-1}$ is a deformation or perturbation of $\mathrm{C} S_{n}$.

First, we explain what we mean about $\mathscr{H}_{n}(t)$ and $\mathrm{C} S_{n}$. The group algebra $\mathrm{C} S_{n}$ is generated by elements $s_{1}, \ldots, s_{n-1}$, where $s_{i}$ denotes the transposition $(i, i+1)$. It has defining relations $(1)_{s},(2)_{s}$ and $s_{i}^{2}=1$. On the other hand, the algebra $\mathscr{H}_{n}(t)$ is generated by $g_{1}^{*}, \ldots, g_{n-1}^{*}$, with defining relations (1) $g^{*},(2)_{g^{*}}$ and $\left(g_{i}^{*}\right)^{2}=(t-1) g_{i}^{*}+t$. In particular, this last relation tells us that $\mathscr{H}_{n}(1)$ is isomorphic to $\mathbf{C S}_{n}$. Even more, we can use similar pictures to describe monomials in $\mathscr{H}_{n}(t)$ and in $C S_{n}$. Recall the geometric definition of a braid in $\S 1$ above as an equivalence class of embeddings of $I^{(n)}$ in $\mathbf{C} \times I$. Similarly, a permutation $\alpha \in S_{n}$ may be regarded as an equivalence class of immersions of $I^{(n)}$ in $\mathbf{R} \times I \subset \mathbf{C} \times I$. See Figure 9. Multiplication is by concatenation and rescaling, as for braids. There are $n$ ! possible equivalence classes of embeddings, and $\mathrm{C} S_{n}$ is the free algebra on these $n$ ! possible patterns.

Pictures similar to those we have just given in $\mathrm{C} S_{n}$ were used by $\mathrm{R}$. Brauer in [Br] to describe another algebra $\mathscr{D}_{n}(x)$ which contains $\mathbf{C} S_{n}$ as a subalgebra. One has the same $2 n$ base points, i.e. $n$ in $\mathbf{R} \times\{0\}$ and $n$ in $\mathbf{R} \times\{1\}$, only now the immersions of $I^{(n)}$ in $\mathbf{R} \times I$ are allowed to join these $2 n$ points in pairs in an arbitrary fashion, that is we no longer require that the arcs join a point 
on $\mathbf{R} \times\{0\}$ to a point on $\mathbf{R} \times\{1\}$. One then obtains a set of $1 \cdot 3 \cdot 5 \cdots(2 n-1)$ pictures because the first point can be joined to $2 n-1$ others, the second to $2 n-3$ others, etc. Multiplication is as before by concatenation, erasure of the middle line and rescaling, but there is a new phenomenon. If $S, T$ are two such graphs, let $U$ be the graph obtained by concatenation and erasure as above. Note that on concatenation there may be closed loops which arise in the middle. Let $m(S, T)$ be the number of such loops. Then $S T=(x)^{m(S, T)} U$, where $x$ is a parameter. The free algebra on the $1 \cdot 3 \cdot 5 \cdots(2 n-1)$ generalized patterns with this product rule, is Brauer's algebra $\mathscr{D}_{n}(x)$. It is easy to see that it is generated by $2 n-2$ elements $s_{1}, \ldots, s_{n-1}, e_{1}, \ldots, e_{n-1}$ where $s_{i}$ and $e_{i}$ are the patterns shown in Figure 9.
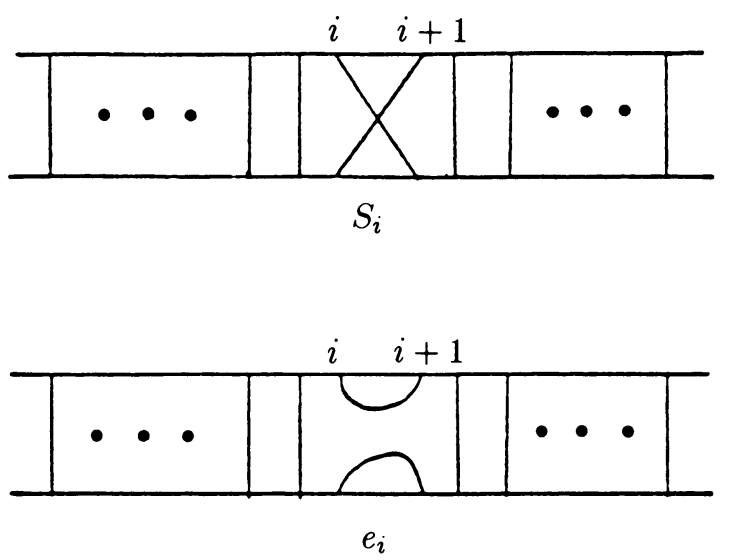

FIGURE 9

Note that for the point $l=i, m=0$ in the parameter space of our algebra $E^{2}$ cannot be determined by relation (21). We will avoid this singularity by choosing different parameters (which results in "blowing up" the point $(i, 0)$ to a line). After these substitutions it will be easy to see how our algebras are connected with Brauer's. So let us define elements $\widehat{G}_{j}, \widehat{E}_{j}$ and parameters $\hat{l}$ and $x$ by

$$
G_{j}=i \widehat{G}_{j}, \quad E_{j}=-\widehat{E}_{j}, \quad l=-i \hat{l} \quad \text { and } \quad m=i \frac{\hat{l}^{-1}-\hat{l}}{x+1}
$$

or, if we solve for $x$

$$
x=m^{-1}\left(l+l^{-1}\right)-1 .
$$

It is immediate that whenever $x$ and $m$ are well defined, the algebra $\hat{\mathscr{C}}_{n}(\hat{l}, x)$ generated by $\widehat{G}_{1}, \widehat{E}_{1}, \ldots, \widehat{G}_{n-1}, \widehat{E}_{n-1}$ is equal to $\mathscr{C}_{n}(l, m)$. Note that relations (13)-(20) also hold for $\widehat{G}_{j}$ and $\widehat{E}_{j}$ instead of $G_{j}$ and $E_{j}$, while (21) becomes $\widehat{E}_{j}^{2}=x \widehat{E}_{j}$. If we set $\hat{l}=1$, we obtain from (22) $\widehat{G}_{j}^{2}=1$. One may now check (by pictures) that $\widehat{G}_{j} \rightarrow s_{j}$ and $\widehat{E}_{j} \rightarrow e_{j}$ can be extended to a homomorphism from $\hat{\mathscr{C}}_{n}(1, x)$ onto $\mathscr{D}_{n}(x)$. Comparing the dimensions, it follows that 
$\hat{\mathscr{C}}_{n}(1, x)$ is isomorphic to $\mathscr{D}_{n}(x)$. So Brauer's algebra can be considered as a special case of our algebra. We finally remark that this does not determine the structure of $\mathscr{D}_{n}(x)$. As we have seen in $\S 4$, the algebras at special values of the parameters can have quite a different structure. The above mentioned problem is studied in a separate paper [W,2] which uses the connections of Brauer's algebra with the representation theory of orthogonal groups.

\section{MATRIX REPRESENTATIONS OF $B_{n}$}

A classical open problem is whether the braid groups $\left\{B_{n} ; n=1,2,3, \ldots\right\}$ are linear groups. For $n=1,2,3$ the answer is known to be "yes", but for $n>3$ the problem is unsolved. Since our work gives new linear representations of $B_{n}$ it seems worthwhile to give some of the new representations explicitly.

There are three mutually nonisomorphic 1-dimensional representations of $C_{2}$ belonging to the idempotents $p_{1}, p_{2}$ and $p_{3}$ as defined in the proof of Theorem 3.7.

We now turn to $B_{3}$. It follows directly from the defining relations that $E_{1}, E_{2} E_{1}$ and $G_{2} E_{1}$ form a basis of a minimal left ideal of $C_{2} E_{2} C_{2}$. Using this basis, we obtain the following new matrix representations of $B_{3}$ :

$$
\sigma_{1} \mapsto\left[\begin{array}{ccc}
l^{-1} & m & 0 \\
0 & m & 1 \\
0 & -1 & 0
\end{array}\right], \quad \sigma_{2} \mapsto\left[\begin{array}{ccc}
0 & 0 & -1 \\
0 & l^{-1} & l^{-1} m \\
1 & 0 & m
\end{array}\right]
$$

Theorem 3.7 determines the structure of $\mathscr{C}_{3}(l, m)$ inductively. See Figure 8, or proceed as follows. We know that

$$
\mathscr{C}_{2} \cong \mathscr{H}_{2} \oplus \mathscr{C}_{1} E_{1} \mathscr{C}_{1} \cong \mathbf{C}(l, \alpha)^{3}
$$

The inclusion matrix $G$ for $\mathscr{C}_{1} \subset \mathscr{C}_{2}$ is a $3 \times 1$ matrix given by

$$
G=\left(\begin{array}{l}
1 \\
1 \\
1
\end{array}\right)
$$

Using (28) and Proposition 3.4, (iii) we obtain that

$$
\mathscr{C}_{2} E_{2} \mathscr{C}_{2} \cong M_{3}(\mathbf{C}(l, \alpha))
$$

This shows that the matrices above generate the full matrix algebra of $3 \times 3$ matrices. On the other hand $\mathscr{H}_{3} \cong \mathrm{C}[l, \alpha] S_{3}$, the group algebra of the symmetric group on 3 letters. Hence

$$
\mathscr{H}_{3} \cong \mathbf{C}(l, \alpha)^{2} \oplus M_{2}(\mathbf{C}(l, \alpha)) .
$$

Similarly, we can determine

$$
\mathscr{C}_{3} E_{3} \mathscr{C}_{3} \cong M_{6}(\mathbf{C}(l, \alpha)) \oplus M_{6}(\mathbf{C}(l, \alpha)) \oplus M_{3}(\mathbf{C}(l, \alpha))
$$

Then $\mathscr{H}_{4}^{\prime}$ is also $\mathscr{C}_{3} E_{3} \mathscr{C}_{3}$, a direct sum of two six-dimensional representations and one 3 -dimensional representation. Since the representations of $B_{4}$ in $\mathscr{H}_{4}$ 
are known to be determined by the Burau representation of $B_{4}$ (see [J,2]), we focus on $\mathscr{H}_{4}^{\prime}$. The 3-dimensional representation of $B_{4}$ factors through $B_{3}$, and so it cannot be faithful, but the 6-dimensional ones stand a chance. We therefore describe one of them in detail.

As above, let $m=\alpha+\alpha^{-1}$, and let

$$
e_{i}=\frac{1}{m^{-1}\left(l+l^{-1}\right)-1} E_{i} .
$$

Let $v=\left(G_{1}-\alpha\right)\left(1-e_{1}\right)$. Then, a basis for the 6-dimensional part which we investigate is $v E_{3}, E_{2} v E_{3}, G_{2} v E_{3}, E_{1} E_{2} v E_{3}, G_{1} E_{2} v E_{3}$, and $G_{1} G_{2} v E_{3}$. Allowing the generators $G_{1}, G_{2}, G_{3}$ of $\psi\left(B_{4}\right)$ to act on this basis on the left, and using the defining relations for $C_{4}$, we obtain the following two parameter matrix representations of $B_{4}$ :

$$
\begin{gathered}
\sigma_{1} \rightarrow\left[\begin{array}{cccccc}
\alpha^{-1} & 0 & 0 & 0 & 0 & 0 \\
0 & 0 & 0 & 0 & -1 & 0 \\
0 & 0 & 0 & 0 & 0 & -1 \\
0 & 0 & 0 & l^{-1} & m l^{-1} & \alpha m l^{-1} \\
0 & 1 & 0 & 0 & m & 0 \\
0 & 0 & 1 & 0 & 0 & m
\end{array}\right], \\
\sigma_{2} \rightarrow\left[\begin{array}{ccccccc}
0 & 0 & -1 & 0 & 0 & 0 \\
0 & l^{-1} & m l^{-1} & m & 0 & 0 \\
1 & 0 & m & 0 & 0 & 0 \\
0 & 0 & 0 & m & 1 & 0 \\
0 & 0 & 0 & -1 & 0 & 0 \\
0 & 0 & 0 & 0 & 0 & \alpha^{-1}
\end{array}\right], \\
\sigma_{3} \rightarrow\left[\begin{array}{ccccccc}
l^{-1} & m & 0 & 0 & \alpha{ }^{-1} & m & 0 \\
0 & m & 1 & 0 & 0 & 0 \\
0 & -1 & 0 & 0 & 0 & 0 \\
0 & 0 & 0 & \alpha^{-1} & 0 & 0 \\
0 & 0 & 0 & 0 & m & 1 \\
0 & 0 & 0 & 0 & -1 & 0
\end{array}\right]
\end{gathered}
$$

We ask the question: Is this representation faithful? By the methods described in [Bi] it would probably suffice to prove that the matrix group generated by $\sigma_{1} \sigma_{3}^{-1}$ and $\sigma_{2} \sigma_{1} \sigma_{3}^{-1} \sigma_{2}^{-1}$ is free of rank 2 .

\section{REFERENCES}

[Al] J. W. Alexander, A lemma on systems of knotted curves, Proc. Nat. Acad. Sci. U.S.A. 9 (1923), 93-95.

[Ar] E. Artin, Theorie der Zopfe, Hamburg Abh. 4 (1925), 47-72.

[Bi] J. S. Birman, Braids, links and mapping class groups, Ann. of Math. Studies, no. 82, Princeton Univ. Press, 1974.

[B-L-M] R. Brandt, W. B. R. Lickorish and K. Millett, A polynomial invariant for unoriented knots and links, preprint. 
[Bo] Claude Bourin, Unpublished notes.

[Br] R. Brauer, On algebras which are connected with the semisimple continuous groups, Ann. of Math. 38 (1937), 857-872.

[FYHLMO] P. Freyd, D. Yetter, J. Hoste, W.B.R. Lickorish, K. Millett, and A. Ocneanu, A new polynomial invariant of knots and links, Bull. Amer. Math. Soc. (N.S.) 12 (1985), 239-246.

[H] J. Hoste, A polynomial invariant of knots and links, Pacific J. Math. 124 (1986), 295-320.

[J,1] V. F. R. Jones, Index for subfactors, Invent. Math. 72 (1983), 1-25.

[J,2] _ _ Braid groups, Hecke algebras and type II factors, Geometric Methods in Abstract Algebras, Proc. U.S.-Japan Symposium, Wiley, 1986, pp. 242-273.

[J,3] _ _ A polynomial invariant for knots via Von Neumann algebras, Bull. Amer. Math. Soc. (N.S.) 12 (1985), 103-111.

[J,4] _ Hecke algebra representations of braid groups and link polynomials, Ann. of Math. 126 (1987), 335-388.

[K,1] L. Kauffman, An invariant of regular isotopy, preprint.

[K,2] _ , A states model for the Jones polynomial, Topology 26 (1987), 395-407.

$[\mathrm{K}, 3] \ldots$, Sign and space: knots and physics, Lecture Notes, Torino.

[Ki] M. Kidwell, On the dimension of the Birman-Wenzl algebra, Unpublished notes.

[La] S. Lang, Algebra, Addison-Wesley, 1965.

[L-M] W. B. R. Lickorish and K. Millett, A polynomial invariant of oriented links, Topology 26 (1987), 107-141.

[Li] W. B. R. Lickorish, A relationship between link polynomials, Math. Proc. Cambridge Philos. Soc. 100 (1986), 109-112.

[Lu] George Lusztig, On a theorem of Benson and Curtis, J. Algebra 71 (1981).

[M] J. Murakami, The Kauffman polynomial of links and representation theory, Osaka J. Math. 24 (1987), 745-758.

[M-S] H. Morton and H. B. Short, Calculating the 2-variable polynomial for knots presented as closed braids, preprint.

[M-T] H. Morton and P. Traczyk, Knots, skeins and algebras, preprint.

[O] A. Ocneanu, A polynomial invariant for knots; a combinatorial and algebraic approach, preprint.

[S] S. Sundaram, On the combinatorics of representations of $S_{p}(2 n ; \mathbf{C})$, Mem. Amer. Math. Soc. (to appear).

[W,1] H. Wenzl, Representations of Hecke algebras and subfactors, Invent. Math. 24 (1988), 349383.

[W,2] __, On the structure of Brauer's centralizer algebras, Ann. of Math. 128 (1988), 179-193.

[Y] David Yetter, Private correspondence with the first author.

Department of Mathematics, Columbia University, New York, New York 10027

Department of Mathematics, University of California, San Diego, la Jolla, CaliforNIA 92093 